SESSION IV: CHEMOTHERAPY

CHAIRPERSON: PROFESSOR JACQUES GROSSET 


\title{
A rapid, microplate-based assay for evaluating the activity of drugs against Mycobacterium leprae, employing the reduction of Alamar Blue
}

\author{
S. FRANZBLAU \\ Institute for Tuberculosis Research, College of Pharmacy, University \\ of Illinois at Chicago, Chicago, IL, USA
}

The nature of the efforts to discover new drugs for the treatment of infectious diseases has changed dramatically in recent years. Both combinatorial chemistry and collections of large numbers of plants have produced huge numbers of interesting compounds, driving the need for high-throughput screening assays. These compounds could be screened for activity against Mycobacterium leprae if high-throughput assays were also available for this organism. However, the existing metabolic screens for anti-leprosy activity in vitro either require too many organisms, or are not adaptable to a microplate format. ${ }^{1-6}$ Described here is the adaptation for $M$. leprae of a microplate-based assay developed originally for determination of the drug-susceptibility of M. tuberculosis. ${ }^{7}$

Oxidation-reduction dyes are non-specific indicators of cellular metabolism, and, as such, can be used to estimate the viability of most bacteria, fungi or animal cells. The best known of these indicators are the tetrazolium dyes, such as MTT. When reduced, the resulting formazans are often water-insoluble, requiring the use of solubilizing reagents if spectrophotometric measurements are to be made.

The Alamar Blue reagent is a proprietary mixture of another redox reagent, resazurin, the structure of which is shown in Figure 1, with stabilizing compounds. This mixture is watersoluble, non-toxic, and non-fluorescent, and is blue in colour. When the N-oxide is reduced, the product, resorufin, which is also water-soluble and non-toxic, fluoresces with excitation and emission maxima of 530 and $590 \mathrm{~nm}$, respectively. Resorufin is red in colour, and absorbs light maximally at $570 \mathrm{~nm}$; however, because the absorption maximum of resazurin is $600 \mathrm{~nm}$, absorption at the latter wavelength must be subtracted from that obtained at $570 \mathrm{~nm}$, thereby reducing the sensitivity of the spectrophotometric assay. On the other hand, because resazurin is not fluorescent, and its presence does not cause appreciable background fluorescence, the fluorometric assay is more sensitive.

The Alamar Blue reagent, used in both a tube ${ }^{8}$ and a microplate format (MABA) $)^{7,9,10}$, as an indicator of growth or inhibition of $M$. tuberculosis, has demonstrated good correlation with the BACTEC 460 and proportion techniques, both for the determination of susceptibility or resistance of the organism to antimicrobials, ${ }^{9,10}$ and in the search for new anti-mycobacterial agents. ${ }^{7}$

As it has been adapted for M. leprae, the MABA makes use of organisms freshly harvested from nude mice, and only partially purified by differential centrifugation. The organisms were incubated at $37^{\circ} \mathrm{C}$ in a Middlebrook-based medium at a density of approximately $10^{7}$ organisms per well in 96-well plates. The cultures were exposed to drugs for 1 or 2 weeks in a gaseous environment of either $2.5 \%$ or ambient oxygen. Following 


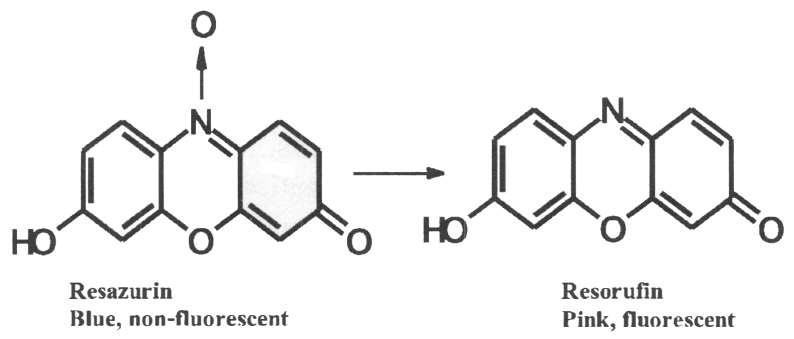

Figure 1. The structural formulae of resazurin and its reduction product, resorufin.

addition of Alamar Blue reagent and Tween 80, and incubation for $24 \mathrm{~h}$, cultures were assessed for reduction of Alamar Blue by determination of fluorescence in a microplate fluorometer. Control cultures (drug-free) typically yielded 10,000-20,000 net fluorescence units (minus the background of heat-killed cultures).

Dose-related responses were observed to all drugs tested. Rifampicin and clarithromycin effected the greatest reductions of activity, followed by minocycline and sparfloxacin. Oflaxacin demonstrated a lesser response, followed by dapsone and clofazimine. The activities observed in the MABA were very similar to those observed previously in radiorespirometric assays. ${ }^{1,11,12}$

The microplate-based assay should permit high-throughput screening of large numbers of compounds for activity against $M$. leprae. The assay requires no processing other than the addition of two reagents, followed by direct measurement of output in a microplate flurometer (or spectrophotometer). Using $M$. leprae only partially purified by differential centrifugation, approximately 1000 assays could be performed from the yield of a single nude mouse.

\section{References}

${ }^{1}$ Franzblau SG. Drug susceptibility testing of Mycobacterium leprae in the BACTEC 460 system. Antimicrob Agents Chemother, 1989; 33: 2115-2117.

${ }^{2}$ Franzblau SG, Biswas AN, Jenner P, Colston MJ. Double-blind evaluation of BACTEC and Buddemeyer-type radiorespirometric assays for in vitro screening of antileprosy agents. Lepr Rev, 1992; 63: 125-133.

${ }^{3}$ Franzblau SG, Harris EB, Hastings RC. Axenic incorporation of $\mathrm{U}^{-14} \mathrm{C}$ palmitic acid into the phenolic glycolipid-1 of Mycobacterium leprae. FEMS Microbiol. Lett, 1987; 48: 407-411.

4 Harris EB, Franzblau SG, Hastings RC. Inhibition of phenolic glycolipid-I synthesis in extracellular Mycobacterium leprae as an indicator of antimicrobial activity. Int J Lepr, 1988; 56: 588-591.

5 Hastings RC, Franzblau SG. Chemotherapy of leprosy. Annu Rev Pharmacol Toxicol, 1988; 28: 231-245.

${ }^{6}$ Hastings RC, Gillis TP, Krahenbuhl JL, Franzblau SG. Leprosy. Clin Microbiol Rev, 1988; 1: 330-348.

7 Collins L, Franzblau SG. Microplate Alamar Blue assay versus BACTEC 460 system for high-throughput screening of compounds against Mycobacterium tuberculosis and Mycobacterium avium. Antimicrob Agents Chemother, 1997; 41: 1004-1009.

8 Yajko DM, Madej JJ, Lancaster MV et al. Colorimetric method for determining MICs of antimicrobial agents for Mycobacterium tuberculosis. J Clin Microbiol, 1995; 33: 2324-2327.

9 Franzblau SG, Witzig RS, McLaughlin JC et al. Rapid, low-technology MIC determination with clinical Mycobacterium tuberculosis isolates by using the microplate Alamar Blue assay. J Clin Microbiol, 1998; 36: $362-366$.

10 Palomino JC, Portaels F. Simple procedure for drug susceptibility testing of Mycobacterium tuberculosis using a commercial colorimetric assay. Eur J Clin Microbiol Infect Dis, 1999; 18: 380-383.

11 Franzblau SG, O'Sullivan JF. Structure-activity relationships of selected phenazines against Mycobacterium leprae in vitro. Antimicrob Agents Chemother, 1988; 32: 1583-1585.

12 Franzblau SG, White KE. Comparative in vitro activities of 20 fluroquinolones against Mycobacterium leprae. Antimicrob Agents Chemother, 1990; 34: 229-231. 


\section{DISCUSSION}

Professor Ji: Why do your results indicate that clarithromycin is almost as bactericidal as rifampicin? This is not the case in mouse experiments.

Professor Franzblau: I don't have a good explanation. On the basis of weight, the drugs are equally active. This situation cannot be easily equated to that in vivo.

Dr Rambukkana: Do you think your assay can be used to determine the viability of M. leprae in tissues?

Professor Franzblau: I think that the radiometric assays are better for this purpose, because they are more sensitive. The Buddemeyer radiometric assay can be used with as few as $10^{6}$ organisms, whereas this assay requires a minimum of $10^{7} \mathrm{M}$. leprae.

Professor Grosset: I am surprised that an intracellular organism like $M$. leprae appears to exhibit metabolic activity in your extracellular cultures. Can you explain this phenomenon?

Professor Franzblau: In our system, the M. leprae are probably dying slowly. The culture conditions appear to maintain metabolism for approximately 2 weeks. We have never seen evidence of multiplication under these conditions. And culturing in the presence of reduced $\mathrm{O}_{2}$ appears to slow the rate at which the organisms die. Enough metabolic activity remains to show the activity of drugs, especially those that block protein synthesis.

Professor Brennan: Does your work employ only M. leprae of the Thai 53 strain? Do organisms of other strains isolated more recently from patients and passed through nude mice behave similarly?

Professor Franzblau: We have not employed organisms of another strain in this assay. However, when we used the radiometric assay to measure the response of 30 to 40 patients to chemotherapy, all of the strains appeared to behave similarly. Although results varied from patient to patient, the range of metabolic activity appeared to be roughly that of the Thai 53 strain, and of a Carville isolate that we had used earlier as our laboratory strain. 